\title{
Coexistence in Molecular Communications
}

\author{
Malcolm Egan*, Trang C. Mai, Trung Q. Duong and Marco Di Renzo
}

\begin{abstract}
Molecular communications is emerging as a technique to support coordination in nanonetworking, particularly in biochemical systems. In complex biochemical systems such as in the human body, it is not always possible to view the molecular communication link in isolation as chemicals in the system may react with chemicals used for the purpose of communication. There are two consequences: either the performance of the molecular communication link is reduced; or the molecular link disrupts the function of the biochemical system. As such, it is important to establish conditions when the molecular communication link can coexist with a biochemical system. In this paper, we develop a framework to establish coexistence conditions based on the theory of chemical reaction networks. We then specialize our framework in two settings: an enzyme-aided molecular communication system; and a low-rate molecular communication system near a biochemical system. In each case, we prove sufficient conditions to ensure coexistence.
\end{abstract}

Keywords: Molecular communications, coexistence, chemical reaction networks.

*Corresponding author.

M. Egan is with the CITI Lab, 69621 Villeurbanne, France which is a joint laboratory in Université de Lyon, INSALyon, and INRIA (e-mail: malcom.egan@inria.fr). T.C. Mai and T.Q. Duong are with the Institute of Electronics, Communications and Information Technology in Queen's University Belfast, Belfast BT7 1NN, U.K. (e-mail: tmai01@qub.ac.uk, trung.q.duong@qub.ac.uk). M. Di Renzo is with the Laboratoire des Signaux et Systémes, CNRS, CentraleSupélec, Univ Paris Sud, Université Paris-Saclay, 91192 Gif-surYvette, France (e-mail: marco.direnzo@12s.centralesupelec.fr). 


\section{INTRODUCTION}

Like any large-scale network, devices that form nanonetworks must be able to perform three basic tasks [1]: sensing; actuation; and coordination. As such, the ability for these nanoscale devices to share information or communicate is a key requirement. In fact, due to the smallscale of nanoscale devices, they are limited in their ability to function in isolation and need to exploit collaborative sensing and actuation, which is only possible when they can communicate.

Due to strict size and energy constraints on the devices that make up a nanonetwork, communication strategies differ from traditional macroscale communication networks. One promising communication strategy is molecular communication, where information is encoded in the number, type or timing of information molecules emitted by a transmitting device [2]. The information molecules then propagate through a fluid medium-often via diffusion - to be received by the intended devices.

There have now been many studies of molecular communication systems from the perspectives of information theory and communication theory [3]. Communication in biological systems has also been explored using tools from systems biology, particularly for quorum sensing in bacteria colonies [4], [5]. These studies include the capacity of molecular communication links [6]-[10], receiver design [11], [12], and inter-symbol interference mitigation strategies [13]. However, a widespread assumption is that molecular communication links exist in an isolated chemical system. That is, there are no other biochemical systems that may interact with the molecular communication link.

Some of the most ambitious proposals for molecular communication are applications within the human body (see e.g., [14]), which consists of a large number of complex biochemical systems. As such, it is not always possible to assume that the molecular communication link will not interact with a pre-existing biochemical system and hence to view the molecular communication link as an isolated system.

There are two potential side effects of this interaction: the molecular communication link's performance may be reduced; and the function of the biochemical system may be disrupted. Clearly, if the biochemical system is, for instance, a cell in the human body it is important to ensure that the impact of the molecular communication link is limited. In this case, the molecular communication link and the biochemical system are said to coexist.

It is worth noting that coexistence in molecular communication bears similarities to coexistence 
in wireless communication networks. In particular, the analogous situation is where an unlicensed wireless network is introduced, which interferes with a pre-existing licensed wireless network. For wireless communication networks, the cognitive radio framework [15] has been proposed to ensure that the operation (or function) of the licensed wireless network is preserved.

However, there is not as yet a framework analogous to cognitive radio for molecular communication links interacting with a pre-existing biochemical system. This is in part due to the fact that the interaction is of a completely different nature. In fact, the interaction between a molecular communication link and a biochemical system is through chemical reactions.

Existing work on the problem of coexistence in molecular communication systems is focused on nanomedicine applications, where the goal is to ensure that the maximum concentration of information molecules introduced by the communication link does not exceed a given level to prevent toxicity [16]. Although the maximum concentration or quantity of information molecules is an important factor, it is also clear that the impact of the molecular communication link on the biochemical system will also depend on the type of molecules used to carry information and the structure of the biochemical system.

In this paper, we instead study the problem of characterizing the impact of a molecular communication link on a pre-existing biochemical system. In particular, we develop a framework to establish conditions when a molecular communication system and a biochemical system can coexist. Our framework is based on the theory of chemical reaction networks [17], which accounts for how the concentrations of different chemical species in a biochemical system evolve over time. This dynamical aspect of our framework is important because the function of a biochemical system depends not only on the maximum concentration of a given chemical species, but also on the evolution of the concentrations. By basing our framework on chemical reaction networks, we are able to study how the evolution of concentrations in the biochemical system changes in the presence of a molecular communication link.

In general, there is not a single definition of coexistence, and it will depend on features of both the molecular communication link and the biochemical system. Using our framework, we address the coexistence problem in two settings, each with their own notion of coexistence. In the first setting, we investigate the effect of introducing enzymes into a molecular communication link for the purpose of reducing inter-symbol interference [13]. In this setting, the coexistence question reduces to how much the concentration of a given chemical species in a pre-existing biochemical system is perturbed with the introduction of the enzymes. We show that it is possible 
to derive closed-form approximations for the time the enzymes are active such that coexistence is possible, which provides insight into the relationship between the concentration of the enzyme and the perturbation of the biochemical system.

In the second setting, we consider the interaction of the information molecules with a wide class of biochemical systems. In particular, we focus on biochemical systems with a unique equilibrium. In this setting, the coexistence question is whether or not the equilibrium of the biochemical system is preserved after introducing a low-rate molecular communication link. Using tools from the theory of chemical reaction networks, we provide explicit conditions on the choice of information molecules to ensure coexistence.

\section{A. Summary of Contributions}

We summarize our contributions as follows:

1) We develop a framework for systems consisting of a biochemical system and a molecular communication link. The framework is based on the theory of chemical reaction networks and accounts for the time-evolution of the concentration of chemical species in the biochemical system.

2) We apply our framework to a system with a molecular communication link that exploits enzymes to reduce inter-symbol interference. We derive closed-form approximations for the perturbation of a biochemical system due to the presence of the enzymes. This provides a means of quantifying the duration of time that the enzymes should be active.

3) We also apply our framework to a system where the information molecules from a low-rate molecular communication link interact with chemical species in a large class of biochemical systems. We derive explicit conditions on the choice of information molecules to ensure that the equilibrium of the biochemical system is preserved. We verify our characterization in an example via numerical solution of the system of ordinary differential equations governing the system.

\section{B. Organization of the Paper}

The remainder of the paper is organized as follows. In Section II, we introduce our model which applies to wide range of biochemical systems and molecular communication links. In Section III, we analyze the first setting where enzymes are used to reduce inter-symbol interference. 
In Section IV, we study how a low-rate molecular communication link affects a biochemical system with a unique equilibrium. In particular, we derive conditions on the information molecules to ensure that the equlibrium is preserved. In Section V, we conclude and discuss open questions.

\section{The REACTION-Network MODEL}

In this section, we develop a framework to model the interactions between a biochemical system and a molecular communication link. We view a biochemical system as a collection of chemical species with time-varying concentrations due to reactions that can take place between the species. As a consequence, our framework is based on chemical reaction network theory. This provides a means of accounting for evolutions of the concentration for each chemical species over time.

\section{A. Modeling Biochemical Systems}

In our model, the biochemical system is a set of chemical species that are related by a set of chemical reactions. As such, the building blocks are the chemical species in the system, the possible reactions between each subset of species, and the rates at which these reactions occur. Before introducing the chemical reaction network model, we begin with the popular example of enzyme-activated biochemical systems [18].

An important class of enzyme-activated biochemical systems consists of four chemical species: the enzyme $E$; the reactant $S$; the complex $\overline{E S}$; and the product $P$. The set of chemical species in this example is then $\mathcal{S}_{E}=\{E, S, \overline{E S}, P\}$. In this system, there are three reactions:

$$
\begin{aligned}
& E+S \stackrel{k_{1}}{\rightarrow} \overline{E S} \\
& \overline{E S} \stackrel{k_{2}}{\rightarrow} E+S \\
& \overline{E S} \stackrel{k_{c q t}}{\rightarrow} E+P,
\end{aligned}
$$

where $k_{1}, k_{2}, k_{\text {cat }}$ are the reaction rate coefficients.

A convenient way of representing each of these reactions is as a map from $\mathbb{N}^{\mathcal{S}_{E}}$ to $\mathbb{N}^{\mathcal{S}_{E}}$. For example, the first reaction is then written as $(1,1,0,0) \rightarrow(0,0,1,0)$. In this way, we can define a set of reactions $\mathcal{R}_{E}=\left\{\mathbf{y}_{i} \rightarrow \mathbf{y}_{i}^{\prime}, i=1,2,3\right\}$, where $\mathbf{y}_{i} \in \mathbb{N}^{\mathcal{S}_{E}}$ is the vector of reactants in reaction $i$ and $\mathbf{y}_{i}^{\prime} \in \mathbb{N}^{\mathcal{S}_{E}}$ is the vector of products.

The pair of chemical species and chemical reactions $\left(\mathcal{S}_{E}, \mathcal{R}_{E}\right)$ is known as a chemical reaction network. In order to model a biochemical system, we also need to consider the dynamics of the 
reactions. Let $[E](t),[S](t),[\overline{E S}](t),[P](t)$ denote the concentration of each chemical species at time $t$. Under the standard assumption of mass-action kinetics [19], the concentrations of each species in the enzyme-activated biochemical system are then governed by the following system of ordinary differential equations

$$
\begin{aligned}
& \frac{d[E](t)}{d t}=-k_{1}[E](t)[S](t)+k_{2}[\overline{E S}](t)+k_{c a t}[\overline{E S}](t) \\
& \frac{d[S](t)}{d t}=-k_{1}[E](t)[S](t)+k_{2}[\overline{E S}](t) \\
& \frac{d[\overline{E S}](t)}{d t}=k_{1}[E](t)[S](t)-k_{2}[\overline{E S}](t)-k_{c a t}[\overline{E S}](t) \\
& \frac{d[P](t)}{d t}=k_{\text {cat }}[\overline{E S}](t),
\end{aligned}
$$

with initial conditions $[E](0)=E_{0},[S](0)=S_{0},[\overline{E S}](0)=\overline{E S}_{0}$, and $[P](0)=P_{0}$. We further assume the conservation law $[E](t)+[\overline{E S}](t)=E_{0}+\overline{E S}_{0}$ holds. The biochemical system can then be written as the tuple $\left(\mathcal{S}_{E}, \mathcal{R}_{E}, k_{E}\right)$, where $k_{E}:\{1,2,3\} \rightarrow\left\{k_{1}, k_{2}, k_{\text {cat }}\right\}$.

We now present the definition of a biochemical system used in the remainder of this work.

Definition 1. A biochemical system is the tuple $(\mathcal{S}, \mathcal{R}, k)$, consisting of a set of chemical species $\mathcal{S}$, a set of reactions $\mathcal{R}=\left\{\mathbf{y}_{i} \rightarrow \mathbf{y}_{i}^{\prime}, i=1,2, \ldots\right\}$, and the rate function $k$.

Let $\mathbf{x}(t) \in \mathbb{R}^{\mathcal{S}}$ be the vector consisting of concentrations of each chemical species at time $t$. Under mass-action kinetics, the dynamics of the biochemical system is governed by

$$
\dot{\mathbf{x}}(t)=\sum_{\mathbf{y} \rightarrow \mathbf{y}^{\prime} \in \mathcal{R}} k_{\mathbf{y} \rightarrow \mathbf{y}^{\prime}} \mathbf{x}(t)^{\mathbf{y}}\left(\mathbf{y}^{\prime}-\mathbf{y}\right),
$$

where $\mathbf{x}(t)^{\mathbf{y}}=x_{1}(t)^{y_{1}} x_{2}(t)^{y_{2}} \cdots$.

This model for biochemical systems is directly applicable under the assumptions of mass balance, constant temperature, constant pressure and a spatially uniform concentration of reactants [20]. Although these conditions are not always present, the reaction network framework provides a tractable way of analyzing the behavior of biochemical systems, which must otherwise be treated on a case-by-case basis informed by experimental data. In order to establish when the reaction network model is applicable, note that in general the concentration of each species is governed by reaction-diffusion equations, given by

$$
\frac{\partial\left[A_{i}\right]}{\partial t}=D_{i} \nabla^{2}\left[A_{i}\right]+R_{i}, \quad i=1,2, \ldots
$$


where $R_{i}$ is the contribution of the reactions to the dynamics and $D_{i}$ is the diffusion coefficient. In general, it is necessary to account for both the diffusion and reaction terms. Nevertheless, the reaction network framework is a good approximation in the reaction-limited regime, where the effect of diffusion is negligible. This regime can be quantified by the Damköhler number $D_{a}$ [21], which is a dimensionless parameter given by the ratio of the characteristic diffusion time to the characteristic reaction time. In particular, when $D_{a} \ll 1$, the reactions dynamics dominate the diffusion dynamics and the reaction network framework can be applied.

As we will see in more detail in Section III and Section IV, reaction networks can be applied to obtain important insights into the coexistence problem. In particular, we are able to consider both quantitative and qualitative features of the complete system including both the molecular communication link and the biochemical system. A key qualitative feature of biochemical reaction systems is the existence of an equilibrium point. In particular, when it exists, an equilibrium point of a biochemical system $(\mathcal{S}, \mathcal{R}, k)$ is defined as $\lim _{t \rightarrow \infty} \mathbf{x}(t)$. The analysis of qualitative features has the desirable aspect that the results are robust in the sense that they do not depend on precise values of parameters nor the form of the reactions [22].

\section{B. Modeling the Molecular Communication Link}

We now turn to modeling molecular communication links within the framework of chemical reaction networks. Consider a transmitting device that emits $K$ chemical species at time $t=0$, with concentrations $\left[I_{j}\right](0)=L_{j}, j=1,2, \ldots, K$. We assume that none of the species $\left\{I_{j}\right\}$ react with each other. Therefore, in the absence of any other chemical species, the only way for the concentrations $\left[I_{j}\right](t), j=1,2, \ldots, K$ to vary is for the receiving device to absorb the information molecules $\left\{I_{j}\right\}$.

Suppose that the rate coefficient for the transmitting device's production of chemical species $I_{j}$ is $k_{I_{j}, p}$ and the rate coefficient for the receiving device absorption of chemical species $I_{j}$ is $k_{I_{j}, a}$. Then, the chemical reactions for the information molecules are

$$
\begin{gathered}
\emptyset \stackrel{k_{I_{j}, p}}{\rightarrow} I_{j} \\
I_{j} \stackrel{k_{I_{j}, a}}{\rightarrow} \emptyset,
\end{gathered}
$$

where $\emptyset$ corresponds to a zero molecule, which arises when either a new molecule of $I_{j}$ is produced or an existing molecule of $I_{j}$ is absorbed. In diffusion-based molecular communication, 
the rate coefficient $k_{I_{j}, a}$ corresponds to the rate that information molecules diffuse from the transmitting device and are absorbed by the receiver.

As such, the molecular communication link can be viewed as a chemical reaction system $\left\{\mathcal{S}_{I}, \mathcal{R}_{I}, k_{I}\right)$, with chemical species $\mathcal{S}_{I}=\left\{I_{1}, \ldots, I_{K}\right\}$, reactions $\mathcal{R}_{I}$ defined in (5), and reaction rate function $k_{I}: j \mapsto k_{I_{j}}$.

Recently, methods to reduce inter-symbol interference have been introduced which modify the reactions in (5). A key example is the enzyme-aided approach in [13]. In this approach, enzymes are introduced into the fluid to reduce inter-symbol interference by reducing the quantity of persisting information molecules. It is straightforward to include such approaches into our model by adding these new chemical species to $\mathcal{S}_{I}$ and reactions to the set $\mathcal{R}_{I}$, which we explore in detail in Section III.

\section{Modeling the Complete System}

We have seen that both the molecular communication link and any pre-existing biochemical system can be modeled as individual chemical reaction networks. In order to establish conditions for these systems to coexist, the remaining step is to form a model for the complete system. In particular, we show how to compose the biochemical system and the molecular communication link to form the model of the complete system.

The first step is to construct the set of chemical species, which is simply the union $\mathcal{S}_{B} \cup \mathcal{S}_{I}$. To obtain the set of reactions, note that it is possible that species in $\mathcal{S}_{B}$ may react with species in $\mathcal{S}_{I}$ resulting in reactions that are neither in $\mathcal{R}_{B}$ nor $\mathcal{R}_{I}$. In this case, it is non-trivial to establish coexistence. Let $\mathcal{R}_{C}$ denote the set of reactions that are neither in $\mathcal{R}_{B}$ nor $\mathcal{R}_{I}$. The resulting chemical reaction system for the complete system is therefore $\left(\mathcal{S}_{B} \cup \mathcal{S}_{I}, \mathcal{R}_{B} \cup \mathcal{R}_{I} \cup \mathcal{R}_{C}, k_{C}\right)$, where $k_{C}$ is the rate function for all reactions in $\mathcal{R}_{B} \cup \mathcal{R}_{I} \cup \mathcal{R}_{C}$.

With a model for the complete system in hand, we now study the coexistence problem. We begin with enzyme-aided molecular communication links in Section III.

\section{ENZYME-AIDED MOLECULAR COMMUNICATIONS}

In molecular communication systems, enzymes have been proposed as means to reduce intersymbol interference. However, in the presence of a biochemical system, these enzymes may in fact catalyze unintended chemical reactions. Although many enzymes are highly specialized, promiscuous enzymes can arise [23] which may not only react with information molecules in a 
molecular communication link, but also with chemical species in the pre-existing biochemical system.

In this section, we study the coexistence problem for enzyme-aided molecular communication links. In particular, we consider a molecular communication link, which includes an enzyme $E$. In addition, there is a biochemical system $\left(\mathcal{S}_{B}, \mathcal{R}_{B}, k_{B}\right)$ containing a single chemical species $S$ that can react with the enzyme $E$ with dynamics governed by the equations in (2). We assume that the biochemical system is at equilibrium-i.e., the concentrations of species in $\mathcal{S}_{B}$ converge as $t \rightarrow \infty$-and that the reaction between $S$ and $E$ occurs at a much faster rate than $S$ with any other species in $\mathcal{S}_{B}$.

The question we are concerned with is to establish how long the enzyme $E$ can be present in order to ensure that the concentration $[S](t)$ does not drop below a threshold $S_{0}-\Delta$ from its initial concentration $S_{0}$. In practice, the enzymes can be switched off using inhibition networks [18] where the molecular communication link introduces additional molecules that bind at a rapid rate to the enzymes, preventing species in the biochemical system from interacting with the enzymes. For biochemical systems that are locally stable, this condition can ensure that the concentrations of species in the biochemical system return to the same equilibrium point.

Formally, we seek the time

$$
t^{*}=\sup \left\{t|| S_{0}-[S](t) \mid \leq \Delta\right\}
$$

Moreover, we say that the molecular communication link and the biochemical system coexist if the enzyme $E$ is not activated after time $t^{*}$. As such, the analysis in this section provides first insights into the influence of coexistence constraints on molecular communication link design.

In order to find the time $t^{*}$, it is in general necessary to solve the equations in (2), which is not possible in closed-form. Nevertheless, these equations can be simplified under the pseudo steady-state assumption (PSS), which holds when $E_{0} \ll K_{m}+S_{0}$, where

$$
K_{m}=\frac{k_{2}+k_{c a t}}{k_{1}}
$$

In particular, the equations under PSS can be well approximated by the Michaelis-Menten kinetics [18, Eq. (2.9)]

$$
\frac{d[S](t)}{d t}=-\frac{k_{c a t}\left(E_{0}+\overline{E S}_{0}\right)[S](t)}{K_{m}+[S](t)} .
$$

The Michaelis-Menten kinetics form a good approximation to the mass action dynamics of the enzyme-activated system in (2) when the PSS property holds and have also been used as a 
model for molecular communication systems in [13]. This equation can be solved in terms of the Lambert W-function as [24, Eq. (27)]

$$
[S](t)=K_{m} W(F(t))
$$

where $W(\cdot)$ is the Lambert $\mathrm{W}$-function given by the solution to

$$
x=W(x) e^{W(x)}
$$

the function $F(t)$ is given by

$$
F(t)=\frac{S_{0}}{K_{m}} \exp \left(\frac{1}{K_{m}}\left(S_{0}-V_{\max } t\right)\right),
$$

and $V_{\max }=k_{c a t}\left(E_{0}+\overline{E S}_{0}\right)$.

By exploiting (9), it is straightforward to solve for $t^{*}$ in (6). However, we can obtain an even simpler expression for $t^{*}$ in the case that $S[t] \gg K_{m}$. Here, from (8), it follows that

$$
\frac{[S](t)}{d t}=-V_{\max }
$$

which implies

$$
S_{0}-[S](t)=V_{\max } t \leq \Delta,
$$

and hence

$$
t^{*}=\frac{\Delta}{V_{\max }} .
$$

To verify the approximation in (14) for the time $t^{*}$, which corresponds to the maximum duration of time the enzyme $E$ can be activated, we investigate the influence of the reaction rate coefficients and the perturbation $\Delta$ numerically in Fig. 1. In the figure, the parameters are $K_{m}=10^{-2}, S_{0}=10^{-2} M, E_{0}=166 \mu M$, with $k_{c a t}$ ranging from $0.5 s^{-1}$ to $10 s^{-1}$, which are consistent with the design of the molecular communication link in [13] and with the parameters for promiscuous enzymes [23]. Observe from the figure that the approximation of $t^{*}$ in (14) is in good agreement with the solution in (9). In this regime, the duration the enzyme can be active while still ensuring coexistence is dependent only on the maximum perturbation $\Delta$, the initial concentration of the enzyme $E_{0}$, and the reaction rate coefficient $k_{c a t}$. 


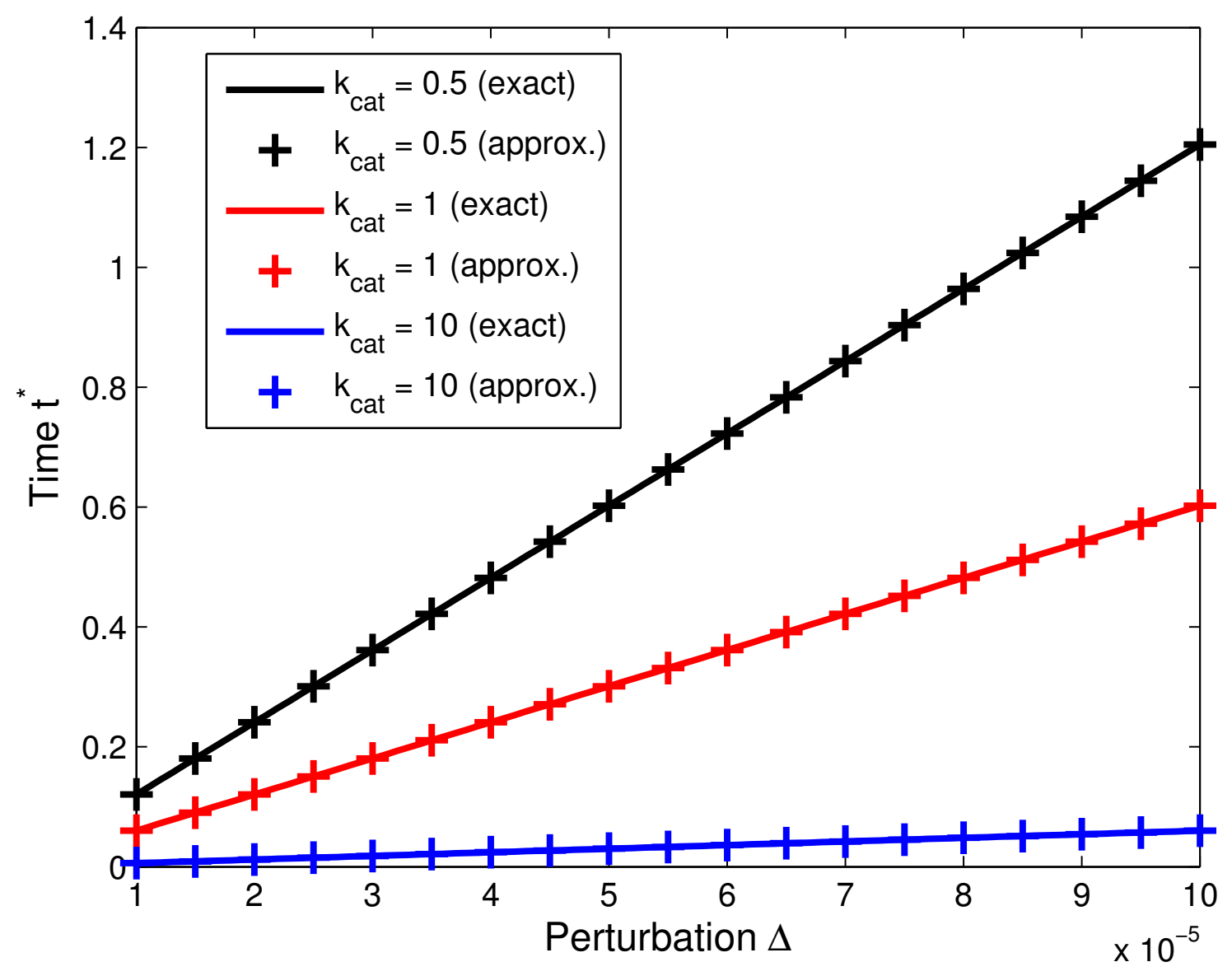

Fig. 1. Plot of the time $t^{*}$ for the enzyme to be activated versus the perturbation $\Delta$ in the concentration of the substrate $S$.

\section{Low-Rate Molecular Communication Links}

In this section, we consider coexistence between a molecular communication link and a large class of biochemical systems. A consequence is that it is no longer possible to obtain closedform approximations for the dynamics of the biochemical system, even without the influence of the molecular communication link. As such, we instead focus on the long-term behavior of the biochemical system in the presence of a low-rate molecular communication link. In particular, we consider a class of biochemical systems with a unique equilibrium and ask the question of how the molecular link should be designed to ensure the equilibrium is preserved.

Characterizing the behavior of a large class of biochemical systems requires more sophisticated use of the chemical reaction network framework. To this end, we begin our analysis by recalling key definitions and a case of the global attractor theorem, which gives conditions for 
a biochemical system to converge to a unique equilibrium.

\section{A. Preliminaries}

Before addressing the coexistence problem for low-rate molecular communication links, we first recall several important definitions and a key result in chemical reaction network theory, which can be found in [25].

The first notion is that of the stoichiometric subspace, which constrains the concentration trajectories. These constraints are induced by the reactions in the system.

Definition 2. The stoichiometric subspace $H \subset \mathbb{R}^{\mathcal{S}}$ is the subspace spanned by $\left\{\mathbf{y}^{\prime}-\mathbf{y} \mid \mathbf{y} \rightarrow\right.$ $\left.\mathbf{y}^{\prime} \in \mathcal{R}\right\}$.

For each initial condition $\mathbf{u}$, the stoichiometric subspace induces a stoichiometric compatibility class, which is given by $(\mathbf{u}+H) \cap \mathbb{R}_{\geq 0}^{\mathcal{S}}$. In particular, the stoichiometric compatibility class is the region of $\mathbb{R}_{\geq 0}^{\mathcal{S}}$ that the concentration trajectories lie in; that is, the valid concentration vectors given the initial condition and the reactions governing the system.

The notion of equilibrium for the biochemical system is formalized in the following definition.

Definition 3. A chemical reaction system $(\mathcal{S}, \mathcal{R}, k)$ with initial concentration vector $\mathbf{u} \in \mathbb{R}^{\mathcal{S}}$ is said to have an equilibrium point $\alpha \in \mathbb{R}^{\mathcal{S}}$ if $\lim _{t \rightarrow \infty} \mathbf{x}(t)=\alpha$. Moreover, let $H$ be the stoichiometric subspace of $(\mathcal{S}, \mathcal{R}, k)$. Then, $\alpha$ is unique if it is the only equilibrium point in $(\mathbf{u}+H) \cap \mathbb{R}_{>0}^{\mathcal{S}}$.

An important class of chemical reaction systems is those that are complex balanced.

Definition 4. A chemical reaction system $(\mathcal{S}, \mathcal{R}, k)$ is complex balanced if there exists a point $\alpha \in \mathbb{R}_{>0}^{\mathcal{S}}$ such that for every $\mathbf{y} \in \mathbb{Z}_{\geq 0}^{\mathcal{S}}$,

$$
\sum_{\mathbf{y} \rightarrow \mathbf{y}^{\prime} \in \mathcal{R}} k_{\mathbf{y} \rightarrow \mathbf{y}^{\prime}} \alpha^{\mathbf{y}}\left(\mathbf{y}^{\prime}-\mathbf{y}\right)=\sum_{\mathbf{z} \rightarrow \mathbf{y} \in \mathcal{R}} k_{\mathbf{z} \rightarrow \mathbf{y} \in \mathcal{R}} \alpha^{\mathbf{z}}(\mathbf{y}-\mathbf{z}) .
$$

The point $\alpha$ is called the point of complex balance.

Intuitively, a point of complex balance corresponds to a concentration vector, where the total rate of production of $\mathbf{y}$ (equivalent to a complex of species) is equal to the total rate of production of complexes in reactions where $\mathbf{y}$ is the substrate, for all $\mathbf{y}$. 
We now turn to establishing conditions on the molecular communication link to ensure that the equilibrium of a nearby biochemical system is preserved.

\section{B. Equilibrium-Preserving Low-Rate Molecular Communication Links}

We now study the coexistence problem for a biochemical system with a unique equilibrium and a low-rate molecular communication link. In particular, we define a low-rate molecular communication link as a one-shot transmission, where information molecules are released only for a finite time and the system ceases to communicate afterwards. From the perspective of the biochemical system, time $t=0$ corresponds to the time that the last information molecule is released by the transmitter. As such, the molecular communication link is modeled as a reaction network with species in $\mathcal{S}_{I}=\left\{I_{1}, \ldots, I_{K}\right\}$ and reactions

$$
I_{j} \stackrel{k_{I_{j}, a}}{\rightarrow} \emptyset
$$

Our main result is a sufficient condition on the choice of information molecules to ensure that the biochemical system preserves its unique equilibrium.

Theorem 1. Let $(\mathcal{S}, \mathcal{R}, k)$ be a weakly reversible complex balanced reaction system with point of complex balance $\alpha$. Suppose that for the trajectory $\mathbf{x}(t)$ with initial conditions $\mathbf{x}(0) \in(\mathbf{u}+$ $H) \cap \mathbb{R}_{\geq 0}^{\mathcal{S}}$, the following limit holds

$$
\lim _{t \rightarrow \infty} \mathbf{x}(t)=\alpha
$$

Further, let $\left(\mathcal{S}_{I}, \mathcal{R}_{I}, k_{I}\right)$ be the reaction system corresponding to a molecular communication link. Suppose that reactions involving species in $\mathcal{S}$ and $\mathcal{S}_{I}$ are of the form

$$
\sum_{j} b_{j} I_{j}+\sum_{j} c_{j} X_{j} \leftrightarrows \sum_{j} d_{j} X_{j}
$$

where at least one element of each set $\left\{b_{j}\right\},\left\{c_{j}\right\},\left\{d_{j}\right\}$ is strictly positive. Then, for any initial conditions $\mathbf{u} \in \mathbb{R}_{>0}^{\mathcal{S}}$, there exists a unique equilibrium in $(\mathbf{u}+H) \cap \mathbb{R}_{\geq 0}^{\mathcal{S}}$ given by $\lim _{t \rightarrow \infty} \mathbf{x}(t)=\alpha$.

Proof. See Appendix A.

Observe from Theorem 1 that sufficient conditions for the molecular communication link to preserve the unique equilibrium of the biochemical system are that the information molecules should be selected to only act as catalyzers and that the reactions between the information molecules and the species in the biochemical system should be reversible. 
The conditions in Theorem 1 place strong constraints on the choice of information molecules. In particular, the conditions suggest that the information molecules should behave like enzymes for chemicals in the biochemical system.

\section{Numerical Results}

To explore the effect of the molecular communication link on a pre-existing biochemical system, we consider the following example. To begin, suppose $\left(\mathcal{S}_{B}, \mathcal{R}_{B}, k_{B}\right)$ is a biochemical system where $\mathcal{S}_{B}=\left\{X_{1}, X_{2}, X_{3}\right\}$ and $\mathcal{R}_{B}$ consists of the reactions

$$
\begin{aligned}
& X_{1}+X_{3} \stackrel{k_{1}}{\rightarrow} 2 X_{2} \\
& 2 X_{2} \stackrel{k_{2}}{\rightarrow} X_{1}+X_{3}
\end{aligned}
$$

It is immediately clear that these reactions are weakly reversible since these reaction are reversible. To verify that the equilibrium is complex balanced, we use the deficiency zero theorem (detailed in Appendix C). The fact that this reaction network converges to a unique equilibrium follows from the global attractor theorem for three-species networks [26].

Further suppose that the effect of the molecular communication link that emits an information molecule $I$ is to introduce the additional reactions

$$
\begin{aligned}
& X_{1}+I \stackrel{k_{3}}{\rightarrow} X_{4} \\
& X_{4} \stackrel{k_{4}}{\rightarrow} X_{1}+I \\
& I \stackrel{k_{L}}{\rightarrow} \emptyset .
\end{aligned}
$$

Observe that the information molecule only acts as a catalyzer and that the new reactions with species in the biochemical system are reversible. As such, Theorem 1 is applicable and the complete system will converge to the same equilibrium as the biochemical system $\left(\mathcal{S}_{B}, \mathcal{R}_{B}, k_{B}\right)$.

To verify the result in the theorem, Fig. 2-4 show the evolution of $X_{1}, X_{2}, X_{3}$ over time for initial concentrations $\left[X_{1}\right](0)=1 M,\left[X_{2}\right](0)=2 M$ and $\left[X_{3}\right](0)=3 M$. Moreover, the molecular communication link introduces $[I](0)=0.05 M$ of information molecules. We also assume that $\left[X_{4}\right](0)=0$. The rate coefficients are $k_{1}=1 M^{-1} s^{-1}, k_{2}=1 s^{-1}, k_{3}=$ $1 M^{-1} s^{-1}, k_{4}=1 s^{-1}, k_{I}=0.1$. In the figures, the unperturbed system corresponds to only the biochemical system, while the perturbed system corresponds to the complete system consisting of both the biochemical system and the molecular communication link. 
Observe in Fig. 2-4 that the equilibrium concentrations for both the biochemical system in isolation and the complete system including the molecular link converge to the same equilibrium, which is consistent with the results in Theorem 1 . However, for small times $t$, the concentrations do not completely agree, which shows that the molecular communication link can in fact impact the behavior of the biochemical system.

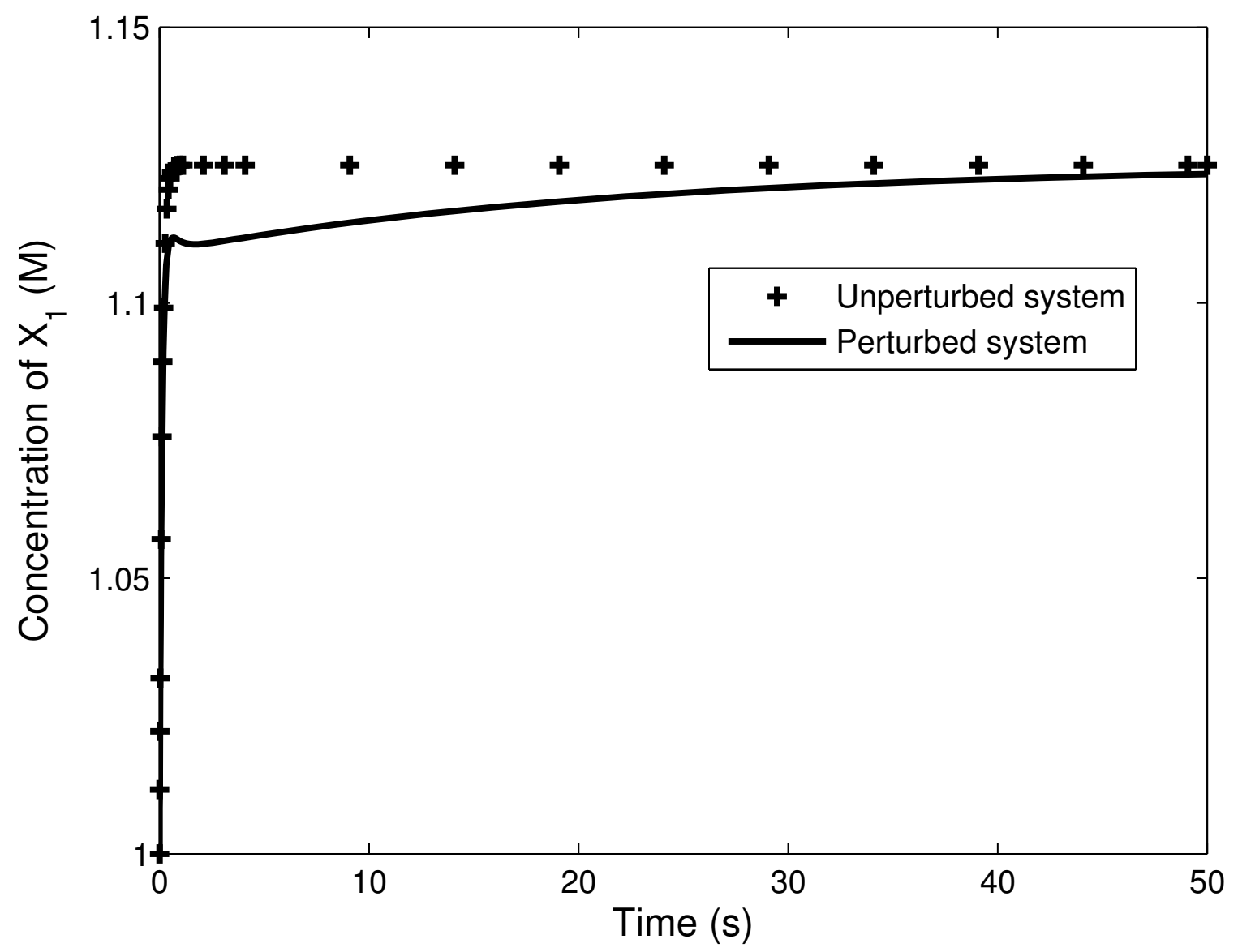

Fig. 2. Plot of the concentration of $X_{1}$ versus time.

\section{Conclusions and Outlook}

If molecular communication links are to fulfil their proposed role in the coordination of nanonetworks embedded in complex biochemical systems, it is crucial that the impact of the links on the biochemical systems is well understood. In other words, conditions under which the molecular communication links and biochemical systems can coexist are required. In this paper, 


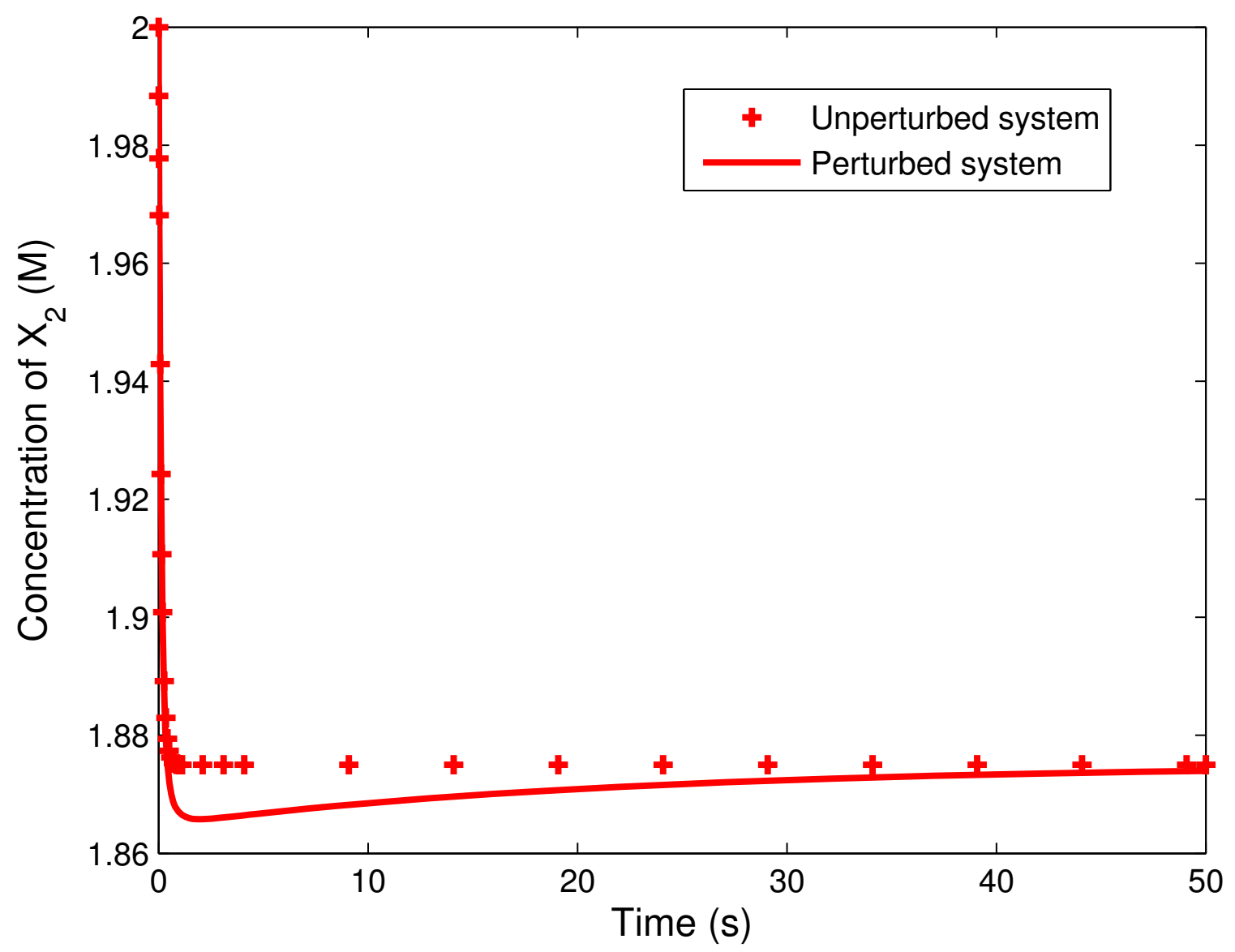

Fig. 3. Plot of the concentration of $X_{2}$ versus time.

we have taken the first steps towards this goal by developing a framework to analyze the effect of a molecular communication link on a biochemical system. Our framework is based on the theory of chemical reaction networks, which provides a basis to consider the time-evolution of the concentrations for chemical species in the biochemical system. This is important in understanding whether the function of the biochemical system is preserved.

We have applied our framework to two special classes of molecular communication links and biochemical systems. In each case, we obtained new constraints on the molecular communication link in order to ensure coexistence with a biochemical system. In dealing with large classes of biochemical systems, it was necessary to exploit general stability results from the theory of chemical reaction networks.

This work raises a number of new questions to ensure that molecular communication links 


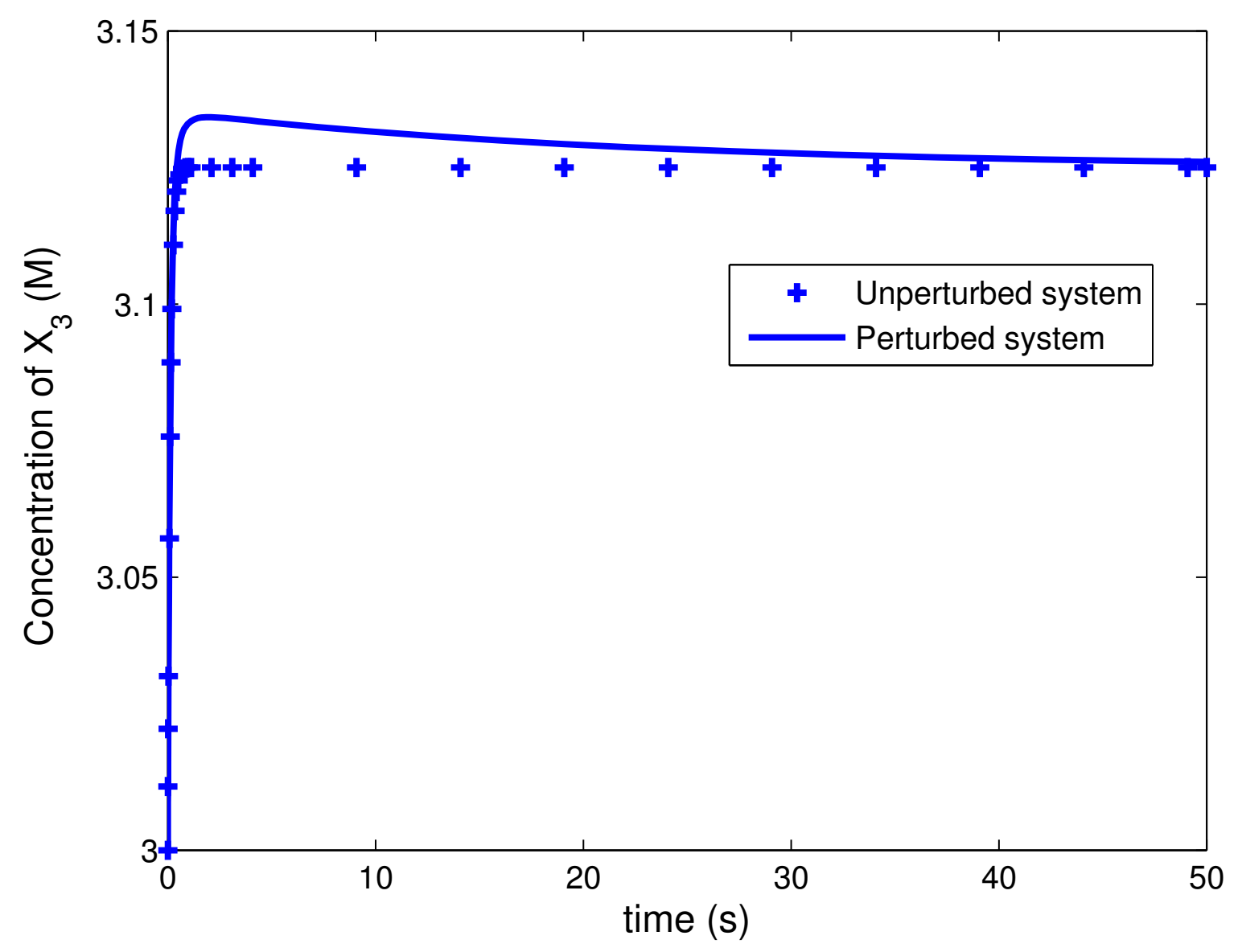

Fig. 4. Plot of the concentration of $X_{3}$ versus time.

can coexist with pre-existing biochemical systems. In particular, can the results in Section IV be generalized to reaction systems with multiple equilibria [20]? A starting point for this question is recent work developing sufficient conditions for multistability in [27]. A related question is whether more complex production and absorption dynamics in the molecular communication link can be accounted for. Recent work in the control literature on advection dynamics [28] has provided results for constant inflows and mass action kinetics outflows, which provide an initial direction to address this question.

Another question is whether or not it is possible to design general purpose molecular communication links that can be embedded in a wide range of biochemical systems? In other words, is it necessary to tailor the design of each molecular communication link to the biochemical system it is embedded in. In the context of enzyme-aided molecular communication, this corresponds 
to the design of the link in order to meet quality of service demands and in particular parameter design which will differ from existing approaches [13], [29].

Another important question is the effect of heterogeneity on the impact of the molecular communication link. In this paper, we have exploited the assumption that the system is well mixed and spatially homogeneous. Does heterogeneity strongly influence the design guidelines for the molecular link? In order to answer this question, it will be necessary to make comparisons with a full reaction-diffusion framework. This can be achieved by simulation studies (e.g., [30]) or a study of qualitative features of reaction-diffusion systems by exploiting recent work in [31], although a full analytical treatment remains challenging as the results in [31] only hold for first order reactions.

\section{APPENDIX A}

\section{PROOF OF THEOREM 1}

Our approach is to view the complete system as a perturbation of the original system. Observe that one of the new reactions only occurs if the required number of information molecules is present. This means that the effective reaction rate of the new reactions is limited by the concentration of information molecules at each time.

Motivated by this observation, we wait for a sufficiently long period of time $t_{1}$ until the concentration of information molecules lies in a $\delta_{1}$-neighborhood of zero, which it will never leave since no new information molecules are produced. By the above observation, the concentration of the species $\left\{X_{1}, X_{2}, X_{3}, \ldots\right\}$ are governed by equations in the differential inclusion

$$
\left\{\sum_{\mathbf{y} \rightarrow \mathbf{y}^{\prime} \in \mathcal{R}} k_{\mathbf{y} \rightarrow \mathbf{y}^{\prime}}^{\prime} \mathbf{x}(t)^{\mathbf{y}}\left(\mathbf{y}^{\prime}-\mathbf{y}\right) \mid k_{\mathbf{y} \rightarrow \mathbf{y}^{\prime}}^{\prime} \in\left(k_{\mathbf{y} \rightarrow \mathbf{y}^{\prime}}-\delta_{1}, k_{\mathbf{y} \rightarrow \mathbf{y}^{\prime}}+\delta_{1}\right)\right\} .
$$

It is then possible to construct a sequence $\delta_{1}>\delta_{2}>\cdots$ corresponding to times $t_{1}<t_{2}<\cdots$ with $\lim _{i \rightarrow \infty} \delta_{i}=0$ and $\lim _{i \rightarrow \infty} t_{i}=\infty$ such that after $t_{i}, \mathbf{x}(t)$ is in a $\delta_{i}$-neighborhood of $\mathbf{x}(\infty)$.

We can now apply the following theorem.

Theorem 2. Let $(\mathcal{S}, \mathcal{R}, k)$ be a weakly reversible complex balanced reaction system with point of complex balance $\beta$ and $\lim _{t \rightarrow \infty} x(t)=\beta$. Fix a point $\mathbf{u} \in \mathbb{R}_{>0}^{\mathcal{S}}$. Consider a sequence $\delta_{1}>$ $\delta_{2}>\cdots>0$ and a sequence $0<t_{1}<t_{2}<\cdots$. Then, $\lim _{t \rightarrow \infty} \mathbf{x}(t)=\beta$.

Proof. See Appendix B. 
The theorem implies that the system will tend to the unique equilibrium point of the underlying biochemical system, as required.

\section{APPENDIX B}

\section{PROOF OF THEOREM 2}

We now prove Theorem 2, which follows a similar argument as [25]. We begin by defining the pseudo-Helmholtz function $g_{\alpha}$.

Definition 5. The pseudo-Helmholtz function $g_{\alpha}: \mathbb{R}_{\geq 0}^{\mathcal{S}} \rightarrow \mathbb{R}$ of $(\mathcal{S}, \mathcal{R}, k)$ at $\alpha$ is the function

$$
g_{\alpha}(x)=\sum_{i \in \mathcal{S}} x_{i} \log x_{i}-x_{i}-x_{i} \log \alpha_{i}
$$

where we take $0 \log 0=0$.

The function $g_{\alpha}$ was shown to be a Lyapunov function for the mass-action equations in (22) by Horn and Jackson [32], which is stated formally in the following theorem.

Theorem 3. If $x$ is a solution to

$$
\dot{\mathbf{x}}(t)=\sum_{\mathbf{y} \rightarrow \mathbf{y}^{\prime} \in \mathcal{R}} k_{\mathbf{y} \rightarrow \mathbf{y}^{\prime}}\left(\mathbf{y}^{\prime}-\mathbf{y}\right) \mathbf{x}(t)^{\mathbf{y}},
$$

with $\mathbf{x}(t) \in \mathbb{R}_{>0}^{\mathcal{S}}$ for all $t \geq 0$ then

$$
\frac{d g_{\alpha}(\mathbf{x}(t))}{d t} \leq 0
$$

with equality if and only if $\mathrm{x}(t)$ is a point of complex balance.

We now prove a preliminary lemma that forms the basis of the proof.

Lemma 1. Let $(\mathcal{S}, \mathcal{R}, k)$ be a weakly reversible complex balanced reaction system with point of complex balance $\beta$. Suppose that for every trajectory $\mathbf{x}(t)$ with initial conditions $\mathbf{x}(0) \in$ $(\mathbf{u}+H) \cap \mathbb{R}_{\geq 0}^{\mathcal{S}}$, the limit

$$
\lim _{t \rightarrow \infty} \mathbf{x}(t)=\beta
$$

Then, for every sufficiently small $\epsilon>0$, there exists $\delta>0$ such that for all $\mathrm{x}^{\prime}$ outside the $\epsilon$-neighborhood of $\beta$ in $(\beta+H) \cap \mathbb{R}_{\geq 0}^{\mathcal{S}}$,

$$
\left.\frac{d\left(g_{\beta}(\mathbf{x}(t))\right)}{d t}\right|_{t=0}<-\delta,
$$


where $\mathbf{x}(t)$ is a solution to the mass-action equations in (22) with $\mathbf{x}(0)=\mathbf{x}^{\prime}$.

Proof. Let $B_{\epsilon}$ be the open $\epsilon$-ball around $\beta$ in $(\beta+H) \cap \mathbb{R}_{\geq 0}^{\mathcal{S}}$ with $\epsilon$ small enough so that $B_{\epsilon}$ does not meet the boundary $\partial \mathbb{R}_{\geq 0}^{\mathcal{S}}$. Define the closed set $K=(\beta+H) \cap \mathbb{R}_{\geq 0}^{\mathcal{S}} \backslash B_{\epsilon}$. Consider $\left.\frac{d g_{\beta}(\mathbf{x}(t))}{d t}\right|_{t=0}$ where $\mathbf{x}(t)$ is a solution to the mass-action equations in (22) with $\mathbf{x}(0)=\mathbf{x}^{\prime}$. Define $\delta=-\inf _{\mathbf{x}^{\prime} \in K} \frac{d g_{\beta}}{d t}\left(\mathbf{x}^{\prime}\right)$. If $\delta \leq 0$ then since $K$ is a closed set and $\frac{d g_{\beta}(\mathbf{x}(t))}{d t}$ is a continuous function, there exists a point $\mathbf{x}^{\prime}$ such that $\frac{d g_{\beta}\left(\mathbf{x}^{\prime}\right)}{d t} \geq 0$, which contradicts Theorem 3 .

We now complete the proof. Fix $\epsilon>0$ such that the $\epsilon$-ball $B_{\epsilon}$ around $\beta$ does not meet the boundary $\partial \mathbb{R}_{\geq 0}^{\mathcal{S}}$. By Lemma 1 , outside $B_{\epsilon}$ there exists $\delta_{\epsilon}>0$ such that $\frac{d g_{\beta}(\mathbf{x}(t))}{d t}<-\delta_{\epsilon}$. Since $\frac{d g_{\beta}(\mathbf{x}(t))}{d t}$ is a continuous function of the rate coefficients $k$, a sufficiently small perturbation $\delta>0$ in the rates will not change the sign of $\frac{d g_{\beta}(\mathbf{x}(t))}{d t}$. Hence, outside $B_{\epsilon}$, the function $g$ is strictly decreasing along $\mathrm{x}(t)$, which implies that eventually every trajectory must enter $B_{\epsilon}$.

Now, for a sequence $\epsilon_{1}<\epsilon_{2}<\cdots$ with $\epsilon_{1}$ sufficiently small such that the $\epsilon_{1}$-ball around $\beta$ does not meet the boundary $\partial \mathbb{R}_{\geq 0}^{\mathcal{S}}$ and $\lim _{i \rightarrow \infty} \epsilon_{i}=0$. From the above argument, it follows that for each $\epsilon_{i}$ there exists a $j$ such that $\delta_{j}$ is sufficiently small and so every trajectory will eventually enter the $\epsilon_{i}$-neighborhood of $\beta$ and never leave. Since this is true for every $i$ and $\lim _{i \rightarrow \infty} \epsilon_{i}=0$, the result follows.

\section{APPENDIX C}

\section{Proof that (19) IS Complex BALANCED}

In order to show that (19) is complex balanced, we require an important result in chemical reaction network theory known as the deficiency zero theorem. Before stating this theorem, we require the concept of the deficiency, which is a number corresponding to the structure of the chemical reaction network. A key parameter in the deficiency is the rank of the network, which is defined as the dimension of the stoichiometric subspace in the network $(\mathcal{S}, \mathcal{R}, k)$.

The deficiency is defined as follows.

Definition 6. The deficiency of a chemical reaction network is defined by

$$
\delta=n-l-s,
$$

where $n$ is the number of chemical complexes, $l$ is the number of connected components in the reaction network, and $s$ is the rank of the network. 
We now can state a restricted form of the deficiency zero theorem [17].

Theorem 4. Suppose we have a chemical reaction network with a finite set of species $\mathcal{S}$ with deficiency zero. Then, if the network is weakly reversible and the rate constants are positive, the mass-action equations have exactly one equilibrium solution in each positive stoichiometric compatibility class. The equilibrium solution is complex balanced.

To show that the chemical reaction system in (19) is complex balanced, we simply need to show that the network has deficiency zero. Observe that there are $n=2$ complexes $\left\{X_{1}+X_{3}, 2 X_{2}\right\}$, $l=1$ connected components, and the rank of the network is $s=1$ since the dimension of $\operatorname{span}((-1,2,-1),(1,-2,1))$ is one. It then follows that $n-l-s=0$ and the deficiency zero theorem applies, so the reaction system is complex balanced.

\section{REFERENCES}

[1] I. Akyildiz, F. Brunetti, and C. Blázquez, “Nanonetworks: a new communication paradigm,” Computer Networks, vol. 52, no. 12 , pp. 2260-2279, 2008.

[2] T. Nakano, A. Eckford, and T. Haraguchi, Molecular Communication. Cambridge University Press, 2013.

[3] F. Farsad, H. Yilmaz, A. Eckford, C.-B. Chae, and W. Guo, "A comprehensive survey of recent advancements in molecular communication,” IEEE Communications Surveys \& Tutorials, vol. 18, no. 3, pp. 1887-1919, 2016.

[4] M. Miller and B. Bassler, "Quorum sensing in bacteria," Annual Reviews in Microbiology, vol. 55, no. 1, pp. 165-199, 2001.

[5] P. Melke, P. Sahlin, A. Levchenko, and H. Jönsson, "A cell-based model for quorum sensing in heterogeneous bacterial colonies," PLoS Comput. Biol., vol. 6, no. 6, 2010.

[6] M. Pierobon and F. Akyildiz, "Capacity of a diffusion-based molecular communication system with channel memory and molecular noise," IEEE Transactions on Information Theory, vol. 59, no. 2, pp. 942-954, 2013.

[7] K. Srinivas, A. Eckford, and R. Adve, "Molecular communication in fluid media: the additive inverse Gaussian noise channel," IEEE Transactions on Information Theory, vol. 58, no. 7, pp. 4678-4692, 2012.

[8] T. Nakano, Y. Okaie, and J.-Q. Liu, "Channel model and capacity analysis of molecular communication with Brownian motion," IEEE Communications Letters, vol. 16, no. 6, pp. 797-800, 2012.

[9] H. Li, S. Moser, and D. Guo, "Capacity of the memoryless additive inverse Gaussian noise channel," IEEE Journal on Selected Areas in Communications, vol. 32, no. 12, pp. 2315-2329, 2014.

[10] M. Egan, Y. Deng, M. Elkashlan, and T. Duong, "Variance-constrained capacity of the molecular timing channel with synchronization error," in IEEE Global Communications Conference (GLOBECOM), 2014.

[11] B. Li, M. Sun, S. Wang, W. Guo, and C. Zhao, "Local convexity inspired low-complexity noncoherent signal detector for nanoscale molecular communications," IEEE Transactions on Communications, vol. 64, no. 5, pp. 2079-2091, 2016.

[12] T. Mai, M. Egan, T. Duong, and M. Di Renzo, "Event detection in molecular communication networks with anomalous diffusion," IEEE Communications Letters, vol. 21, no. 6, pp. 1249-1252, 2017.

[13] A. Noel, K. Cheung, and R. Schober, "Improving receiver performance of diffusive molecular communication with enzymes," IEEE Transactions on NanoBioscience, vol. 13, no. 1, pp. 31-43, 2014. 
[14] B. Atakan, O. Akan, and S. Balasubramiam, "Body area nanonetworks with molecular communications in nanomedicine," IEEE Communications Magazine, vol. 50, no. 1, 2012.

[15] S. Haykin, "Cognitive radio: brain-empowered wireless communications," IEEE Journal on Selected Areas in Communications, vol. 23, no. 2, pp. 201-220, 2005.

[16] Y. Chahibi and I. Akyildiz, "Molecular communication noise and capacity analysis for particulate drug delivery systems," IEEE Transactions on Communications, vol. 62, no. 11, pp. 3891-3903, 2014.

[17] M. Feinberg, "Chemical reaction network structure and the stability of complex isothermal reactors-i. the deficiency zero and deficiency one theorems," Chemical Engineering Science, vol. 42, no. 10, pp. 2229-2268, 1987.

[18] S. Reed, Essential Physiological Biochemistry. Wiley-Blackwell, 2009.

[19] V. Chellaboina, S. Bhat, W. Haddad, and D. Bernstein, "Modeling and analysis of mass-action kinetics," IEEE Control Systems Magazine, vol. 29, no. 4, 2009.

[20] G. Craciun and M. Feinberg, "Multiple equilibria in chemical reaction networks: I. the injectivity property," SIAM J. Appl. Math., vol. 65, no. 5, pp. 1526-1546, 2005.

[21] H. Fogler, Elements of Chemical Reaction Engineering. Prentice Hall, 2006.

[22] D. Angeli, P. Leenheer, and E. Sontag, "A Petri net approach to the study of persistence in chemical reaction networks," 2006.

[23] A. Babtie, N. Tokuriki, and F. Hollfelder, "What makes an enzyme promiscuous?" Current Opinion in Chemical Biology, vol. 14, no. 2, pp. 200-207, 2010.

[24] S. Schnell and C. Mendoza, "Closed form solution for time-dependent enzyme kinetics," J. Theor. Biol., vol. 187, pp. 207-212, 1997.

[25] M. Gopalkrishnan, "A scheme for molecular computation of maximum likelihood estimators for log-linear models," arXiv:1506.03172.

[26] G. Craciun, F. Nazarov, and C. Pantea, "Persistence and permanence of mass-action and power-law dynamical systems," SIAM J. Appl. Math., vol. 73, no. 1, pp. 305-329, 2013.

[27] I. Otero-Muras, P. Yordanov, and J. Stelling, "Chemical reaction network theory elucidates sources of multistability in interferon signaling," PLoS Comput. Biol., vol. 13, no. 4, 2017.

[28] A. van der Schaft, S. Rao, and B. Jayawardhana, "A network dynamics approach to chemical reaction networks," International Journal of Control, vol. 89, no. 4, 2016.

[29] H. Yilmaz, Y.-J. Cho, W. Guo, and C.-B. Chae, "Interference reduction via enzyme deployment for molecular communication,” Electronics Letters, vol. 52, no. 13, pp. 1094-1096, 2016.

[30] A. Noel, K. Cheung, R. Schober, D. Makrakis, and A. Hafid, "Simulating with AcCoRD: actor-based communication via reaction-diffusion," Nano Communication Networks, vol. 11, pp. 44-75, 2017.

[31] K. Fellner, W. Prager, and B. Tang, "The entropy method for reaction-diffusion systems without detailed balance: first order chemical reaction networks," Kinetic and Related Models, vol. 10, no. 4, pp. 1055-1087, 2017.

[32] F. Horn and R. Jackson, "General mass action kinetics," Arch. Rational Mech. Anal., vol. 49, pp. 81-116, 1972. 